\title{
Implementation and Verification of a HELIAS module for the Systems Code PROCESS
}

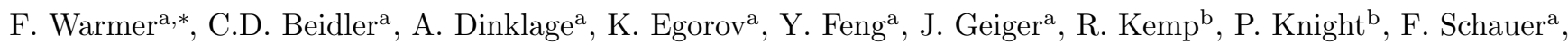 \\ Y. Turkin ${ }^{\mathrm{a}}$, D. Ward ${ }^{\mathrm{b}}$, R. Wolf ${ }^{\mathrm{a}}$, P. Xanthopoulos ${ }^{\mathrm{a}}$ \\ ${ }^{a}$ Max Planck Institute for Plasma Physics, D-17491, Greifswald, Germany \\ ${ }^{b}$ Culham Centre for Fusion Energy, Abingdon, Oxfordshire, OX14 3DB, United Kingdom
}

\begin{abstract}
In order to study design points of next-step fusion devices such as DEMO, comprehensive systems codes are commonly employed. The code package PROCESS is such a tool, widely used for tokamak systems studies. In this work, the implementation and verification of a HELIAS module into PROCESS is addressed. These HELIAS models include: a plasma geometry model based on Fourier coefficients, a basic island divertor model, as well as a coil model which combines scaling aspects based on the Helias 5-B reactor design in combination with analytic inductance and field calculations. The models are verified firstly with respect to W7-X. Secondly, the generality of the models is used to represent the tokamak which is compared against the original tokamak PROCESS models using a DEMO design as reference case. Both approaches show very good agreement.
\end{abstract}

Keywords: HELIAS, PROCESS, Systems Code, verification

\section{Introduction}

Systems codes are simplified, yet comprehensive models of an 35 entire fusion power plant used to carry out respective systems studies. These studies focus on the analysis of the complex 5 interplay between physics, engineering, and economic considerations allowing assessment of parametric dependencies on the design of the plant. The goal of systems studies and systems ${ }_{40}$ codes is the development and optimisation of design points for next-step fusion devices. With this approach critical research 10 areas can be identified. This ansatz is commonly applied in the tokamak community, especially with respect to a tokamak demonstration fusion power plant, also known as 'DEMO', for ${ }_{45}$ which many studies are ongoing.

The systems code PROCESS has been assessed to identify 15 changes necessary to accommodate helical advanced stellarators (HELIAS). Based on this assessment, HELIAS-specific models have been developed in [1] designed for a systems code ${ }_{50}$ approach consisting of three major models. First, a geometry model to describe the plasma shape (flux surfaces) based on

20 Fourier coefficients. Second, a basic island divertor model for the energy exhaust is derived from geometrical considerations, in addition assuming cross-field transport and radiation at the $\mathrm{X}$-point. And third, a coil model which calculates the maximal field at the coils, the total stored magnetic energy, and

25 the dimensions of the winding pack based on the sophisticated 55 Helias 5-B 2] reactor design. For this purpose scaling relations and analytic inductance and field calculations are employed in combination with a critical current density scaling of the superconducting material used.

30 Moreover, a strategy for a predictive confinement time scal- 60 ing has been discussed in [1] where it is shown that 3D turbulence, gyrokinetic GENE simulations have been started for

\footnotetext{
*Corresponding author, Tel.: +49 (0)3834 88-2583

Email address: Felix.Warmer@ipp.mpg.de (F. Warmer)
}

HELIAS geometries [3] with the aim of assessing the behaviour of e.g. ion-temperature-gradient instabilitites. The guiding results can be combined with well-established neoclassical considerations [4] allowing to carry out predictive transport simulations to derive corresponding confinement times which may be compactly employed in systems codes. This development is underway. Meanwhile, stellarator-specific empirical confinement time scalings like ISS04 [5] are available and can be used within PROCESS.

In this work progress is reported on the implementation of the HELIAS models into PROCESS [6] and also the verification of the models with respect to two test cases. In section 2 the architecture of PROCESS and the implementation of the HELIAS models are briefly reviewed. The verification of the HELIAS module is then discussed in section 3 in two parts. First, the models are compared against the stellarator Wendelstein 7-X design and predictions for its performance and second, the generality of the models is used to represent the tokamak and in turn assessed with respect to the original PROCESS tokamak models using a DEMO design as reference case. The work is summarised and the results discussed in section 4

\section{PROCESS Architecture}

PROCESS is a well-established, partly modular, European tokamak systems code which gained maturity through years of applications. A solver based on Lagrangian multipliers is employed within PROCESS to allow for design optimisation with respect to the descriptive models and constraints. This is done by minimising (or maximising) a user-defined Figure of Merit consistent with the relevant inputs (iteration variables, constraint equations, and limits). The framework of PROCESS consists of detailed, well-developed plasma physics, engineering and economic models allowing for a broad scope of application.

65 The modularity of PROCESS also allows implementation options for different confinement concepts. This means, the 
HELIAS models for the plasma geometry, island divertor, and modular coils could be straightforwardly implemented with only minor adaptations. This allows also to retain the use of 70 the non-device-specific systems in PROCESS, such as the balance of plant and economic models, and the numerical solver ${ }_{115}$ which allows constrained optimisation of the design.

\section{Verification of the HELIAS Models}

In order to verify the stellarator module a twofold compar- ${ }^{120}$ 75 ison is carried out. First, the stellarator module is applied to represent Wendelstein 7-X and the results are compared to the W7-X design and predictions for its performance. Second, the generality of the models allows modification of the stellarator module so that it can represent the tokamak. This toka-

80 mak representation of the stellarator module is then compared against a tokamak DEMO reference design point created by the orginal tokamak PROCESS models.

\subsection{Comparison to Wendelstein 7-X}

\subsubsection{Plasma Geometry}

85 The plasma geometry model [1] is based on Fourier coefficients which allows one to describe arbitrarily complex fluxsurfaces. For the validation study the W7-X high-mirror configuration is chosen and the specific Fourier coefficients are obtained from the corresponding VMEC [7] equilibrium. The 90 geometrical parameters of the plasma major radius, plasma minor radius, plasma volume and surface area calculated by the plasma geometry model are in very good agreement with the VMEC results, yielding for both $R=5.5 \mathrm{~m}, a=0.53 \mathrm{~m}$, $S=120 \mathrm{~m}^{2}$ and $V=30.1 \mathrm{~m}^{3}$.

\subsubsection{Island Divertor}

The model of the island divertor concept [1, 8, consists of a geometrical description including cross-field diffusion and radiation around the X-point. For the verification with W7-X, experimental data are not yet available. Therefore, the island divertor model is compared against a 3D EMC3-Eirene [9] simulation of a W7-X high-power discharge scenario. For the EMC3 simulation a heating power of $P=10 \mathrm{MW}$ and perpendicular heat diffusion coefficient of $\chi_{\perp}=1.5 \mathrm{~m}^{2} / \mathrm{s}$ have been chosen. In accordance with W7-X and the simulation results the following values were selected as additional input for the island divertor model: the inclination of the divertor plate relative to the field lines $\alpha_{\lim }=2^{\circ}$, the temperature in front of the divertor plates $T_{\mathrm{t}}=15 \mathrm{eV}$, the radiation fraction in the SOL $f_{\text {rad }}=0.05$, and the field line pitch angle $\Theta=O\left(10^{-3}\right)$. The EMC3 simulation

\begin{tabular}{lcc} 
& EMC3 & ID Model \\
\hline Island Size $[\mathrm{cm}]$ & 14 & $14^{*}$ \\
X-point - target distance $[\mathrm{cm}]$ & 12.5 & $12.5^{*}$ \\
Divertor plate Length $[\mathrm{m}]$ & $1 \sim 1.5$ & 1.6 \\
Power Decay Width $[\mathrm{cm}]$ & 7.4 & 9.3 \\
Effective wetted area $\left[\mathrm{m}^{2}\right]$ & $1 \sim 2$ & 1.5 \\
Heat load $\left[\mathrm{MW} / \mathrm{m}^{2}\right]$ & 6.5 & 6.9 \\
\hline
\end{tabular}

Table 1: Comparison of the important parameters of an EMC3Eirene simulation of Wendelstein $7-\mathrm{X}$ with the corresponding output from the island divertor model. The values marked with a star have here been used as inputs to the island divertor model.
It can be seen from the EMC3 simulation results in Fig. 1] that the energy deposition has a 3D pattern, e.g. long tail in the front of the plate. This means that the parameters defined in the island divertor model cannot be straightforwardly represented by a single value. Especially for the effective length of the wetted area of the divertor, only a range of values can be given (Tab. 1). The value for the effective wetted area calculated by the island divertor model lies within this range and the value for the divertor plate length at the boundary of the EMC3 range. The power decay width and the heat load agree to the right order of magnitude but show a discrepancy of about $20 \%$.

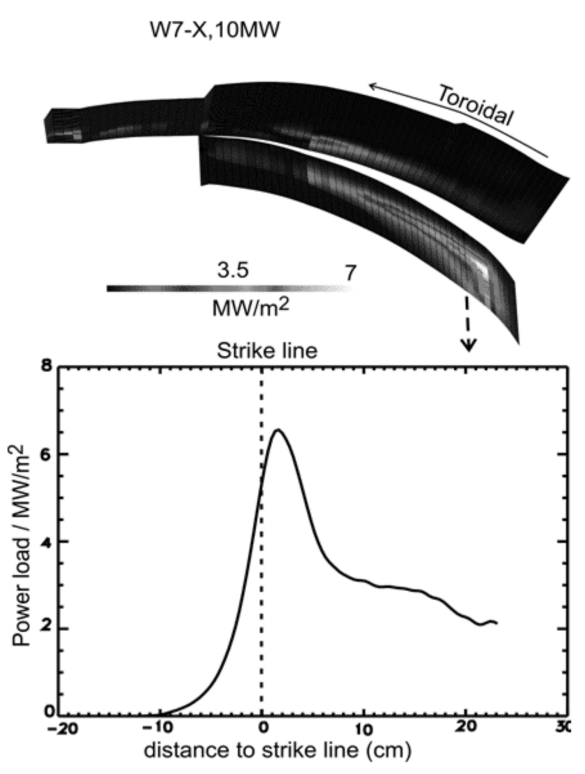

Figure 1: Heat load distribution on a W7-X divertor plate for an EMC3-Eirene simulation and perpendicular cut of the divertor plate front for $P=10 \mathrm{MW}$ and $\chi_{\perp}=1.5 \mathrm{~m}^{2} / \mathrm{s}$.

Contributing to this discrepancy is the fact that the basic island divertor model assumes two stellarator-symmetric targets in each field period, while in reality there are four in W7-X. Although the island divertor model does not take such details into account the first comparison results are encouraging. This preliminary result suggests that the island divertor model provides an acceptable estimation of the heat load within the frame of a systems code but nedds to be justified in a future work.

\subsubsection{Modular Coils}

The model for the modular coils 1 is a combination of analytical calculations and scaling relations which are based on the detailed Helias 5-B coil design employing $\mathrm{Nb}_{3} \mathrm{Sn}$ as superconductor. To be able to compare the coil model with the existing W7-X coils a scaling for the critical current density of NbTi was implemented. This was done in the same fashion as for $\mathrm{Nb}_{3} \mathrm{Sn}$. Apart from that, the size has been scaled to represent the geometrical parameters of W7-X. The results are compared in Tab. 2,

Agreement is found between the self-consistent model and the values for W7-X except for the estimated masses. The mass of the support structure is calculated from an empirical scaling which goes over several orders of magnitude. This scaling gets 45 closer to the virial limit at high magnetic energy representing 


\begin{tabular}{lcc} 
& W7-X & Coil Model \\
\hline Field on Axis [T] & 3.0 & $3.0^{*}$ \\
Field on Coil [T] & 6.7 & 6.6 \\
Magnetic Energy [MJ] & 620 & 640 \\
Mass of Sup. Struc. [t] & $\sim 300$ & 212 \\
Winding pack [mm $\times \mathrm{mm}]$ & $166 \times 226$ & $167 \times 177$ \\
Ampere Turns [MA] & 1.74 & 1.74 \\
Total weight of WP [t] & $\sim 100$ & 62 \\
Average Coil Length [m] & 8.5 & 8.5 \\
\hline
\end{tabular}

Table 2: Comparison of the important parameters of the coil design of Wendelstein 7-X with the corresponding output of the coil model. The value marked with a star has been used as input.

the necessarity for mass optimisation. In W7-X minimisation of the mass of the support structure was not attempted and it ${ }_{190}$ therefore deviates from the model

Also the winding pack aspect ratio is more radially elongated for the W7-X coils which introduces a discrepancy for the winding pack dimensions between W7-X and the coil model as well as for the winding pack mass. In addition, the electrical insula- ${ }_{195}$ tion and winding pack embedding require relatively more cross section in $\mathrm{W} 7-\mathrm{X}$ than in a reactor coil.

Next-step HELIAS devices will have very high stored magnetic energy requiring much more support structure, meaning that optimisation with respect to minimal mass will play a role. 200 Therefore, it is expected that the mass calculations will have better validity for extrapolation of larger devices closer to Helias 5-B while the other parameters are expected to retain their very good agreement.

\subsection{Application to Tokamak-DEMO: a test-case}

In order to further verify the stellarator module, the corresponding models are applied to an axisymmetric tokamak case and the results are compared to the original tokamak PROCESS module. For this study an advanced tokamak DEMO design point was selected as reference case with a major radius $R_{0}=7.95 \mathrm{~m}$, minor radius $a=2.9 \mathrm{~m}$, plasma elongation $\kappa=1.78$ and plasma triangulartiy $\delta=0.5$.

\subsubsection{Plasma Geometry}

The axisymmetric plasma shape in the tokamak PROCESS geometry model is described by two intersecting circles, from which the plasma volume, surface and cross-sectional area can be obtained with simple analytic formulas.

175 As the stellarator geometry model is based on Fourier coefficients, a magnetic equilibrium has been created with VMEC closely representing the tokamak DEMO design point. The toroidal cut for the PROCESS tokamak DEMO shape and the VMEC magnetic equilibrium are both shown in Fig. 22 It can be seen that the created VMEC equilibrium properly resembles ${ }_{210}$ the tokamak PROCESS shape.

A comparison of the important geometrical parameters, namely the plasma volume, the surface area and the crosssectional area for the PROCESS tokamak shape yields very good agreement within $1 \%$ (Tab. 3). Subsequently, the VMEC 21 tokamak DEMO equilibrium has been read in and processed by the stellarator geometry model yielding very good agreement for the plasma volume and the cross-sectional area.

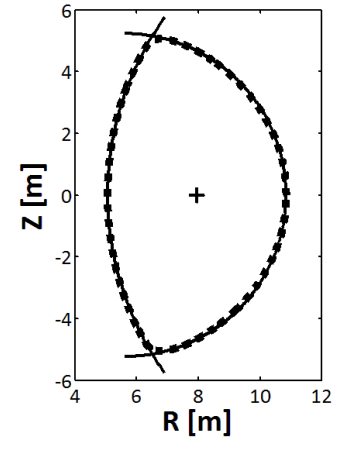

Figure 2: Comparison of the PROCESS DEMO cross-section consisting of two circular arcs (solid lines) with the corresponding VMEC equilibrium (squares).

\subsubsection{Coil Model}

While the generality of the geometry model allowed a straightforward application and comparison with the tokamak DEMO reference case, the stellarator coil model is more specific. Although the analytic part of the coil model is general, the scaling relations are based on the Helias 5-B reactor design which hardly makes a direct application to tokamaks meaningful. Since the coil model is a combination of analytical calculations and scalings based on the Helias 5-B design, it is possible to replace the scaling part with an available tokamak design. For this basis, ITER has been chosen while the analytic part of the coil model is kept unchanged.

With ITER as basis, an 'adapted' coil model can be employed to recreate the tokamak DEMO reference case, comparing it with the original PROCESS results. In order to model the DEMO reference case, the machine geometrical parameters (e.g. major radius, minor radius, shield and blanket width) were adjusted accordingly as well as the dimensions of the individual turns. The corresponding results for the coil dimensions and associated field parameters are shown in Tab. 4

\begin{tabular}{lcc} 
& DEMO & Coil Model \\
\hline Field on Axis [T] & 5.2 & $5.2^{*}$ \\
Field at Coil [T] & 12.9 & 13.2 \\
Magnetic Energy [GJ] & 111 & 113 \\
Winding Pack [m $\times \mathrm{m}]$ & $0.88 \times 0.91$ & $0.82 \times 0.98$ \\
Ampere Turns [MA] & 12.8 & 12.8 \\
Conductor mass per coil [t] & 132.5 & 127.8 \\
Case mass per coil [t] & 520.7 & 509.5 \\
Average Coil Length [m] & 46.6 & 47.3 \\
\hline
\end{tabular}

Table 4: Comparison of the important parameters of the PROCESS DEMO reference case with the corresponding output from the adapted coil model (the PROCESS trapezoidal toroidal winding pack form has been averaged for simplicity). The value marked with a star has been used as input.

It is found that the adapted coil model yields results very similar to those of the original PROCESS model with a maximal relative difference of less than $10 \%$ which is well within the accuracy of such a systems code approach. The main difference arises for the winding pack dimensions which have a different radial to toroidal aspect ratio which is fixed in the adapted coil model based on ITER and more flexible for the original PROCESS model. Overall the adapted coil model shows very good agreement with the PROCESS tokamak DEMO reference case establishing confidence for the use of the model. 


\begin{tabular}{lccc} 
& $\begin{array}{c}\text { PROCESS } \\
\text { representation }\end{array}$ & $\begin{array}{c}\text { VMEC } \\
\text { equilibrium }\end{array}$ & $\begin{array}{c}\text { Stellarator Geometry } \\
\text { Model }\end{array}$ \\
\hline Plasma Volume $\left[\mathrm{m}^{3}\right]$ & 2131 & 2117 & 2117 \\
Plasma Surface Area $\left[\mathrm{m}^{2}\right]$ & 1231 & 1217 & 1235 \\
Cross-Sectional Area $\left[\mathrm{m}^{2}\right]$ & 43.7 & 43.5 & 43.5 \\
\hline
\end{tabular}

Table 3: Comparison of the Plasma Volume, Surface Area and Cross-Sectional Area of the DEMO PROCESS representation with the correspondingly created VMEC equilibrium and the HELIAS Geometry model.

\subsubsection{Divertor Plausibility Check}

The island divertor is conceptually different from the tokamak axisymmetric divertor. Nevertheless, the island divertor model makes use of a basic geometrical approach which, with270 minor modifications, can be adopted to represent the tokamak. The major physical differences between the two concepts are the scales of the connection lengths and the magnetic field pitch angle. In the stellarator the connection lengths are an order of magnitude longer, $L_{c} \sim O\left(10^{3}\right) \mathrm{m}$, than in comparable toka-275 maks, $L_{c} \sim O\left(10^{2}\right) \mathrm{m}$, while the field pitch on the other hand is much smaller in stellarators, $\Theta \sim O\left(10^{-3}\right)$, compared to tokaparallel SOL transport is dominant while in stellarators also the perpendicular transport plays an important role compet-280 ing with parallel transport. Taking additionally into account that the tokamak divertor is toroidally closed, the island divertor model can be modified accordingly. The heat load is given as [1:

$$
q_{\mathrm{div}}=\frac{P_{\mathrm{div}}}{A_{\mathrm{eff}}}=\frac{P_{\mathrm{SOL}}\left(1-f_{\mathrm{rad}}\right)}{L_{\mathrm{T}} \cdot \lambda_{q}} .
$$

The total length of the available divertor is for the tokamak modified to $L_{T} \approx 2 \cdot 2 \pi R$ and the geometric flux expansion,

$240 f_{x} \approx 1 / \alpha_{\text {pol }}[10$, is now considered within the power width on the divertor plates: $\lambda_{q}=f_{x} \cdot \sqrt{\chi_{\perp} \cdot \tau_{\|}}$.

Considering the DEMO reference case, the distance from X-290 point to divertor plate is fixed to $\Delta=0.4 \mathrm{~m}$ and a perpendicular heat diffusion coefficient of $\chi_{\perp}=1 \mathrm{~m}^{2} / \mathrm{s}$ is assumed. In adthe values for $\alpha_{p o l}=20^{\circ}, T_{t}=10 \mathrm{eV}$ and $\Theta=0.1$ have been estimated for this case. Applying the modified model, the power width on the divertor for the tokamak DEMO reference case becomes then $\lambda_{q}=4 \mathrm{~cm}$ yielding an effective wetted area295 of $A_{\text {eff }}=4 \mathrm{~m}^{2}$. The DEMO case is designed for $2 \mathrm{GW}$ fupower resulting in about $400 \mathrm{MW}$ transport power which cross the separatrix. In order to keep the heat load below $q_{\text {div }} \leq 5 \mathrm{MW} / \mathrm{m}^{2}$, it is found in the model that only $5 \%^{300}$ (20 MW) of this power is allowed to reach the divertor.

These results agree on the order of magnitude with values found in e.g. [10] where the divertor broadening for a $\mathrm{DEMO}_{30}$ case is anticipated to be on the order of $1-3 \mathrm{~cm}$ at temperatures below $10 \mathrm{eV}$.

\section{Conclusions}

HELIAS models have been successfully implemented in the module has been carried out. First, W7-X was modeled within the HELIAS-representation of PROCESS and compared to the real machine parameters. The comparison showed very good agreement for the plasma geometry and the coil model, except ${ }^{32}$ the coil masses which could be traced back to the fact that the W7-X support structure is not optimised with respect to mass. Also the island divertor model showed agreement on the dimensions with an EMC3-Eirene simulation with deviations on the order of $20 \%$.

Secondly, the generality of the HELIAS-module has been used to represent the tokamak where the coil module has been adopted using ITER paramters as basis. Moreover, the divertor model was modified taking into account the tokamak symmetry. The subsequent modelling of a DEMO reference case showed very good agreement for the plasma geometry model and the coil model within this representation compared to the original PROCESS tokamak models. Also the modified divertor model gives plausible results agreeing with values found in [10.

With this tool available, stellarator systems studies can be conducted. Moreover, a direct comparison between tokamak and stellarator DEMO and power plant designs can be carried out within the common framework of PROCESS which is subject to ongoing investigations.

\section{Acknowledgments}

This work has been carried out within the framework of the EUROfusion Consortium and has received funding from the European Union's Horizon 2020 research and innovation programme under grant agreement number 633053. The views and opinions expressed herein do not necessarily reflect those of the European Commission.

\section{References}

1] F. Warmer, C. D. Beidler, A. Dinklage et al. "HELIAS Module Development for Systems Codes." Fusion Engineering and Design, vol. [submited] (2014)

[2] F. Schauer, K. Egorov and V. Bykov. "HELIAS 5-B magnet system structure and maintenance concept." Fusion Engineering and Design, vol. 88, p. 1619 (2013).

[3] P. Xanthopoulos, F. Merz, T. Grler et al. "Nonlinear Gyrokinetic Simulations of Ion-Temperature-Gradient Turbulence for the Optimized Wendelstein 7-X Stellarator." Physical Review Letters, vol. 99, p. 035002 (2007).

[4] Y. Turkin, C. D. Beidler, H. Maaßberg et al. "Neoclassical transport simulations for stellarators." Physics of Plasmas, vol. 18, p. 022505 (2011)

[5] H. Yamada, J. H. Harris, A. Dinklage et al. "Characterization of energy confinement in net-current free plasmas using the extended International Stellarator Database." Nuclear Fusion, vol. 45, p. 1684 (2005).

[6] M. Kovari, R. Kemp, H. Lux et al. "PROCESS: A systems code for fusion power plants - Part 1: Physics." Fusion Engineering and Design, vol. [in Press] (2014).

[7] S. P. Hirshman, W. I. van Rij and P. Merkel. "Three-dimensional free boundary calculations using a spectral Green's function method." Computer Physics Communications, vol. 43, p. 143 (1986).

[8] Y. Feng. "Up-scaling the island divertor along the W7-stellarator line." Journal of Nuclear Materials, vol. 438, p. S497 (2013).

[9] D. Reiter, M. Baelmans and P. Börner. "The EIRENE and B2EIRENE Codes." Fusion Science and Technology, vol. 47, p. 172 (2005).

[10] B. Sieglin. Experimental Investigation of Heat Transport and Divertor Loads of Fusion Plasma in All Metal ASDEX Upgrade and JET. Ph.D. thesis, Technische Universität München (2014). 